ADVERSE DRUG REACTION

\title{
Thrombotic thrombocytopenic purpura associated with statin treatment
}

\author{
F Sundram, P Roberts, B Kennedy, S Pavord
}

Postgrad Med J 2004;80:551-552. doi: 10.1136/pgmj.2003.017178

Thrombotic thrombocytopenic purpura (TTP) is a rare condition but associated with $90 \%$ mortality if left untreated. The diagnosis is usually made when there is thrombocytopenia and microangiopathic haemolytic anaemia, although the full pentad also includes fever, renal impairment, and neurological dysfunction. A variety of underlying causes have been implicated in acquired TTP including bacterial and viral infections, bone marrow and organ transplantation, pregnancy, immune disorders, and certain drugs. To date there is just one case report of TTP associated with statin treatment. The clinical course of a patient who presented with TTP after being started on simvastatin, a HMG-CoA inhibitor, is described.

A 68 year old white man was seen in the accident and emergency department with a four hour history of left sided chest pain, which developed while mowing his lawn. He had a previous myocardial infarction in 1989 and was on regular aspirin $75 \mathrm{mg}$ daily. Examination was unremarkable except for an audible systolic murmur at the apex. Initial blood tests were normal, as were the electrocardiogram and chest radiograph. He was diagnosed with angina, received enoxaparin $150 \mathrm{mg}$ subcutaneously, and was discharged the next morning to take oral amlodipine $5 \mathrm{mg}$ and simvastatin $20 \mathrm{mg}$ daily.

The next day he experienced nausea and headache. He discontinued his medication but over the ensuing 48 hours developed widespread bruising, bleeding in the mouth and pain and swelling in the left knee, left ankle, right elbow, and shoulder joints. He had no prior or family history of similar illness and was convinced that the medications had poisoned him, and relayed this to the medical staff on admission to hospital two days later. On examination, he was mildly confused, had a temperature of $39^{\circ} \mathrm{C}$, with normal blood pressure and pulse. He was markedly icteric and had diffuse petechiae over his lower limbs with an effusion in his left knee joint.

Investigations revealed a platelet count of $7 \times 10^{9} / 1$, haemoglobin $112 \mathrm{~g} / \mathrm{l}$, reticulocyte count $75 \times 10^{9} / \mathrm{l}$, and numerous schistocytes on the blood film (see fig 1). Lactate dehydrogenase was $1039 \mu / \mathrm{l}$, bilirubin $100 \mu \mathrm{mol} / \mathrm{l}$, and haptoglobin $<0.3$. Direct Coombs' test was negative and renal function normal. Prothrombin time, activated partial thromboplastin time, and fibrinogen levels were normal but D-dimers were $60000 \mathrm{ng} / \mathrm{ml}$ (normal range: 0-300 ng/ml). Bone marrow morphology showed reactive changes with normal megakaryocytes, consistent with peripheral destruction of platelets.

Management consisted of broad spectrum intravenous antibiotics and daily plasma exchange through a femoral line, with normalisation of his platelet count by the fifth day (see fig 2) and considerable improvement in his clinical condition. However on the third day he sustained an acute coronary syndrome, confirmed by a raised troponin-T level of $1.5 \mu \mathrm{g} / \mathrm{l}$ with $3 \mathrm{~mm}$ depression of ST segments on electrocardiography. This was managed with tinzaparin, atenolol, and subsequently aspirin. It is likely that the anaemia (haemoglobin $86 \mathrm{~g} / \mathrm{l}$ ) and recent plasma exchange contributed to the event. His hospital stay was also complicated by an episode of staphylococcal femoral line sepsis which developed despite broad spectrum antibiotics and prophylactic dalteparin. This was treated with teicoplanin. Plasma exchanges were discontinued after five days but in view of his persistent low grade fever and the systolic murmur, he remained on antibiotics until transoesophageal echocardiogram confirmed no evidence of infective endocarditis. Subsequent tests also showed negative results for blood cultures, leptospira, cytomegalovirus, hepatitis A, B and C, and HIV. Before discharge, he was started on atenolol $50 \mathrm{mg}$ daily for treatment of hypertension but advised to avoid statin medication. Six months later, he remains symptom-free with normal laboratory tests.

\section{DISCUSSION}

Our patient developed symptoms one day after starting simvastatin and TTP was diagnosed when he was admitted four days later. No other underlying illness was found and there was rapid recovery after discontinuation of the drug. These features suggested to us the possibility of drug induced TTP.

Statins have become commonly prescribed drugs in the treatment of hyperlipidaemia. Simvastatin has been proven to significantly reduce fasting low density lipoprotein cholesterol levels, ${ }^{1}$ although it has been associated with serious side effects, including rhabdomyolysis and liver dysfunction. A number of drugs have been implicated in the pathogenesis of TTP (box 1).

There has only been one previous case report involving simvastatin, ${ }^{10}$ however, it is interesting to note that in five of the 11 reported cases of clopidogrel induced TTP, the patient was concurrently taking a statin. ${ }^{11}$ Amlodipine could also

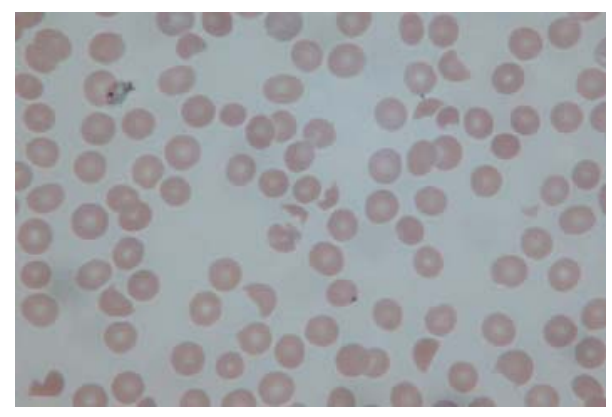

Figure 1 Peripheral blood film in TTP: numerous schistocytes with low platelet numbers. 

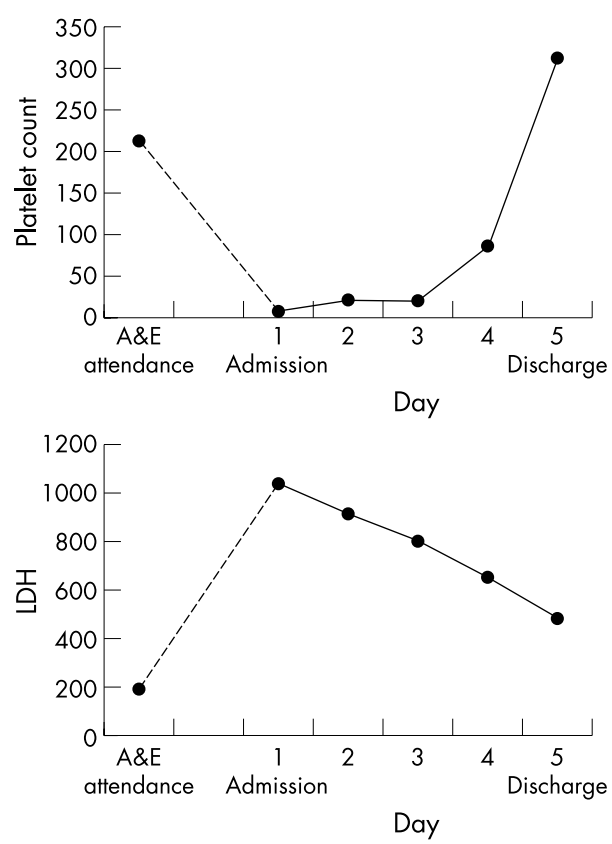

Figure 2 Variation of platelet count and lactate dehydrogenase (LDH) before and during admission (A\&E, accident and emergency).

have been implicated, although no previous association with TTP has been suggested.

His condition improved without the need for continuing total plasma exchanges, perhaps more suggestive of drug induced TTP than that related to sepsis, which appears to undergo temporary exacerbation after the introduction of antibiotics. The timing of the disease makes an IgE immune mediated mechanism unlikely and the associated fever, oedema and arthralgia, resembling serum sickness, suggests a drug hypersensitivity reaction or immune complex mediated reaction related to cross reactivity to previous antigen exposure. We considered the possibility of heparin induced thrombocytopenia but the clinical criteria for this were not met and the platelet count continued to improve despite further heparin treatment.

Although the patient had a good response to plasma exchange, it may have contributed to the acute coronary syndrome, emphasising the need for caution with this mode of treatment. We recognise that this case does not prove a causal relationship between simvastatin and the generation of TTP but find the circumstantial evidence compelling. Furthermore, clinicians should be alerted to the possible association as undiagnosed and untreated TTP leads to death in $>90 \%$ of cases.

\section{Box 1: Drugs associated with TTP}

- Ticlopidine. $^{2}$

- Clopidogrel. ${ }^{3}$

- Quinine. ${ }^{4}$

- Cyclosporin. ${ }^{5}$

- Pentostatin. ${ }^{6}$

- Levornogestrol. ${ }^{7}$

- Dipyridamol. ${ }^{8}$

- Valcyclovir. ${ }^{9}$

\section{Authors' affiliations \\ F Sundram, P Roberts, B Kennedy, S Pavord, Department of \\ Haematology, Leicester Royal Infirmary, Leicester, UK}

Correspondence to: Dr S Pavord, Department of Haematology, Leicester Royal Infirmary, Infirmary Square, Leicester LE1 5WW, UK; sp59@le.ac. uk

Submitted 15 November 2003

Accepted 15 January 2004

\section{REFERENCES}

1 Scandinavian Simvastatin Survival Study Group. Randomised trial of cholesterol lowering in 4444 patients with coronary heart disease: The Scandinavian Simvastatin Survival Study (4S). Lancet 1994;344:1383-9.

2 Page $Y$, Tardy B. Thrombotic thrombocytopenic purpura related to ticlopidine. Lancet 1991;337:1219.

3 Bennett CL, Connors JM, Carwile JM, et al. Thrombotic thrombocytopenic purpura associated with clopidogrel. N Engl J Med 2000;342:1773-7.

4 Gottschall Jl, Neahring B, McFarland JG, et al. Quinine-induced immune thrombocytopenia with haemolytic uraemic syndrome: clinical and serological findings in nine patients and review of the literature. Am J Hematol 1994;47:283-9.

5 Holler $\mathbf{E}$, Kolb HJ, Hiller E, et al. Microangiopathy in patients on cyclosporine prophylaxis who developed acute graft versus host disease after HLA-identical bone marrow transplantation. Blood 1989;73:2018-24.

6 Leach JW, Pham T, Diamandidis D, et al. Thrombotic thrombocytopenic purpua-hemolytic uraemic syndrome (TTP-HUS) following treatment with deoxycoformycin in a patient with cutaneous T cell lymphoma (Sezary syndrome): a case report. Am J Hematol 1999;61:268-70.

7 McShane PM, Bern MM, Schiff I. Thrombotic thrombocytopenic purpura associated with oral contraceptives: a case report. Am J Obstet Gynecol 1983; 145:762-3

8 Lindquist JR, George RP. Thrombotic thrombocytopenic purpura: onset during dipyridamole therapy. JAMA 1981;246:2577.

9 Rivaud E, Massiani MA, Vincent F, et al. Valcyclovir hydrochloride therapy and thrombotic thrombocytopenic purpura in a HIV-infected patient. Arch Intern Med 2000;160:1705-6.

10 McCarthy L, Porcu P, Fausel CA, et al. Thrombotic thrombocytopenic purpura and simvastatin. Lancet 1998;352:1284-5.

11 Paradiso-Hardy FL, Papastergiou J, Lanctot KL, et al. Thrombotic thrombocytopenic purpura associated with clopidogrel: further evaluation. Can J Cardiol 2002; 18:771-3. 